\title{
Artes de pesca y realidades económicas en torno a comunidades de pescadores en el embalse el guajaro.
}

\author{
Rafael Fernando Oyaga Martínez ${ }^{\mathrm{a}}$, Pedro Jessid Pacheco Torres ${ }^{\mathrm{a}}$, Sofía Brisseth de Jesús Sulbaran Siado ${ }^{\mathrm{b}}$ \\ $\&$ Yolbetis Saavedra Benedetti ${ }^{a}$ \\ ${ }^{a}$ Facultad de Ciencias, Administrativas y Contables, Corporación Universitaria Reformada, Barranquilla, \\ Colombia. \\ r.oyaga@unireformada.edu.co,p.pacheco@unireformada.edu.co,ysaavedra@unireformada.edu.co \\ ${ }^{b}$ Facultad de Ingeniería, Universidad de la Costa, Barranquilla, Colombia. \\ sofiasulbaran1117@gmail.com
}

Recibido: Agosto 14, 2018.

Recibido en su versión corregida: Septiembre 16, 2019.

Aceptación: Septiembre 26, 2019.

Cómo citar: Oyaga Martinez, R.F., Pacheco Torres, P.J., Sulbaran Siado, S.B.J. \& Saavedra Benedetti, Y. (2019). Artes de pesca y realidades económicas en torno a comunidades de pescadores en el embalse El Guajaro. Revista Sextante, 21, pp. 5 - 11, 2019.

\section{Resumen}

Se presenta el trabajo de investigación desarrollado con comunidades de pescadores en el embalse El Guajaro entre los años 2013 a 2016, con el fin de determinar los tipos de arte de pesca que implementan en el sector y cómo estos determinan la relación costo - beneficio en las ganancias económicas y en la mejora de la calidad de vida de los habitantes.

Se reporta que existe un preocupante uso de artes de pesca restringidas en este medio, debido al peligro de capturar organismos muy pequeños. En ese sentido hace falta un mayor control por parte de las autoridades. Por otro lado, se remarca por parte de las comunidades de los pescadores, la frustración en cuanto a los beneficios económicos obtenidos por dicha actividad. Se logró determinar que este, en promedio, es inferior al salario mínimo legal vigente, ya que deben repartir las ganancias entre los participantes de la faena y pagar el alquiler de lanchas.

Palabras clave: Actividad pesquera; Calidad de vida; Economía; Embalse.

\section{Fishing gear and economic realities around fishing communities in El Guajaro reservoir.}

\begin{abstract}
The research work carried out with fishing communities in the El Guajaro reservoir between 2013 and 2016 is presented to determine the fishing gear types that they implement in this sector and how it is determined the costbenefit ratio in the economic gains and in improving the inhabitant's life quality.

It is worrying to use restricted fishing gear in this environment, due to the danger of capturing very small organisms. In that sense, greater control is required by the authorities. On the other hand, the frustration regarding the economic benefits obtained by this activity is highlighted by the fishermen's communities. It was possible to determine that is lower than the current legal minimum wage, since they must distribute the profits among the participants of the task and pay the boat rental.
\end{abstract}

Keywords: Economy; Fishing activity; Life quality; Reservoir. 


\section{Introducción}

En Colombia la actividad pesquera en cuerpos de agua lenticos del norte del país se centra fundamentalmente en la pesca artesanal, liderada por hombres de mediana edad, con poco apoyo tecnológico y que dependen fundamentalmente de su experiencia (Garcia, 2010). Esta situación se ve afectada por componentes como: una baja producción relacionada fundamentalmente por la afectación fisicoquímica de los cuerpos de agua, y situaciones asociadas al uso inadecuado de las artes de pesca que se desarrollan en la zona. (Herrera y Cortez 2012).

Por otra parte, los autores destacan en su documento el reducido proceso de investigación sobre el tema por parte de los entes universitarios de la región, y la situación académica de los pescadores. La pesca, como actividad económica que se desarrolla en el embalse El Guajaro, representa el sustento para un grupo importante de familias de corregimientos como: Rotinet (Repelón), La Peña (Sabanalarga). El cuerpo de agua en mención ha venido presentando los siguientes problemas (Oyaga, 2013): Descarga de aguas servidas de los municipios y corregimientos circundantes, la inadecuada disposición de basuras, la práctica de caza y pesca indiscriminada, control de insectos con agroquímicos (especialmente la descarga de plaguicidas organoclorados). El autor también destaca situaciones como la deforestación de las zonas aledañas al embalse. Otra fuente más que afecta al sitio son las aguas provenientes del Canal del Dique, dado que llegan con un alto contenido de sólidos.

La zona llamada Las Compuertas, al mantenerse cerrada, altera significativamente la condición natural del embalse de la siguiente forma: dificulta el lavado de la sal que pueda acumularse, corta el equilibrio biológico de las especies migratorias y no permite la salida de sedimentos. Por otro lado, el arrastre de sólidos provenientes de las canteras de material triturado y la falta de una educación ambiental en las comunidades aledañas complementan las alteraciones.

\section{Antecedentes en la literatura}

Se reporta la construcción de un manual para la gestión ambiental de la pesca artesanal y las buenas prácticas pesqueras en la cuenca del río Paraná, Argentina, en donde se establece que esta labor se debe extender por encima de la actividad pesquera o de los procedimientos de captura. Involucra otros actores y a todos los aspectos relacionados con el desenvolvimiento y el soporte de la pesca propiamente dicha, así como los procesos de postcaptura, procesamiento y mercadeo.

En Chile (Marín, 2007) se describe la necesidad de un cambio del régimen pesquero tradicional a uno con regulaciones, para la protección de los ecosistemas. Los pescadores artesanales deben formalizar su trabajo y cumplir con lo que establece la ley, lo que responde a medidas de gestión de recursos hidrobiológicos y a la regulación impulsada por el Gobierno chileno en 1991.

En Argentina se describió en el año 2000, por parte del Instituto Nacional Pesquero (INIDEP), las diferentes artes de pesca que se practican en la zona del canal de Beagle, en donde se destacan los usos de nasas, trampas de pulpo, palangre de fondo, redes de fondo y redes de enmalle.

En Colombia la Autoridad Nacional de Pesca (AUNAP) estableció que la actividad pesquera se puede agrupar en dos grandes sectores: industrial y artesanal. En el caso de la pesca artesanal, generalmente se realiza con embarcaciones pequeñas, con una limitada autonomía. Igualmente reportan que estos procesos se llevan a cabo manualmente, lo que implica un gran desgaste físico y una relación de beneficio negativa (AUNAP, 2014).

Un documento desarrollado por AUNAP, Artes y métodos de pesca en el año 2014, establece que en la Costa Atlántica la pesca es fundamentalmente artesanal, en donde se destacan artes de pesca como: de malla, anzuelo, arpón y trampas. De otra parte, destacan pesquerías denominadas industriales, como, por ejemplo: la pesca de arrastre de camarón de aguas someras y la pesca palangrero de grandes pelágicos (atún, dorado, etc.), las cuales operan principalmente desde Tolú y Cartagena. 


\section{Metodología}

En la Figura 1 exponemos la metodología que será utilizada durante la investigación.

Se realizó un levantamiento intensivo de la información con las comunidades de pescadores en el embalse y se aplicaron encuestas de observación directa para medir el impacto de las estrategias aplicadas en el cuerpo de agua. También se buscó información en diferentes centros de documentación y bibliotecas de instituciones públicas y privadas de los departamentos del Atlántico y Bolívar, entre ellas la Universidad del Atlántico, la Corporación Universitaria de la Costa (CUC), la Corporación Autónoma Regional del Atlántico (CRA), la Corporación Autónoma Regional del Dique (CARDIQUE), y las gobernaciones de los departamentos en mención. Luego se categorizó la información.

Se realizaron encuestas en los corregimientos de la Peña y Rotinet, evaluando aspectos como estados de los servicios públicos, tipos de artes de pesca utilizadas, número de capturas de peces por día y políticas aplicadas por la Gobernación del Atlántico y la CRA.

La información recolectada fue validada estadísticamente con el programa Spss. En este proceso se buscó reflexionar sobre qué ha pasado en los procesos de investigación en la zona del sur del Atlántico.

\section{Resultados y discusión}

De acuerdo con la metodología presentada anteriormente, se realizará la interpretación de los de datos recolectados en donde se evidenciarán los resultados obtenidos de los tipos de arte de pesca utilizada en el embalse. Figura 2

El arte de pesca más utilizada es la atarraya, debido a condiciones tradicionales que se afincaron desde décadas atrás; en el segundo lugar encontramos que la técnica del trasmallo, en sus diferentes connotaciones, sin importar las consecuencias que para la actividad puede generar el uso de esta práctica. Entre las artes prohibidas de pesca hallamos una representación de $8 \%$. Figura 3

Dentro del desarrollo se alcanzaron a tipificar los peces con los que los pescadores tenían relación, estos son: barbul, corbinata, arenca, trucha arcoiris, cachama, bagre pintado, tilapia roja, mojarra dorada, mojarra lora, bocachico, entre otros.

Se logró identificar que la mojarra lora es el pez que más se extrae del embalse, seguida de la tilapia roja y la arenca, considerando que las siembras de estos peces son las más características de los programas realizados por la CRA y la Gobernación del Atlántico. Figura 4

La tendencia del $49 \%$ de pesca es de 6 a 30 peces diarios extraídos, teniendo en cuenta que sea temporada de alta presencia de estos en el sector; el $34 \%$ realiza pesca entre los 31 a 100 peces diarios y una minoría del $12 \%$ llegan a manejar cantidades entre los 101 a los 1000 peces diarios. Figura 5

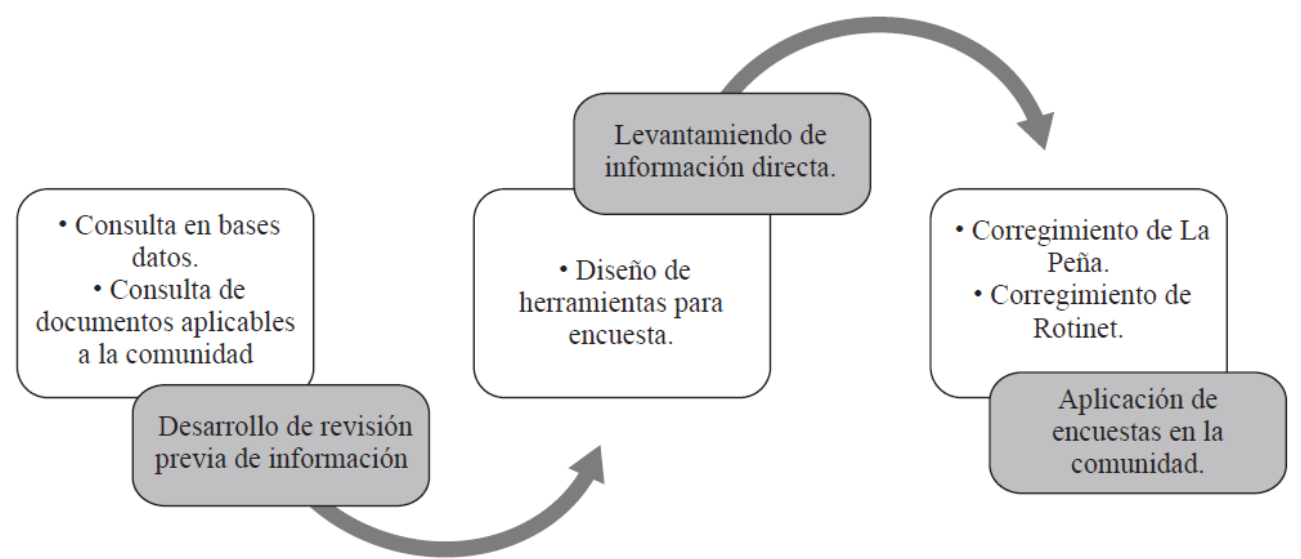

Figura 1. Metodología de estudio.

Fuente: Los autores. 
Oyaga Martínez et al. (2019). Revista Sextante, 21, pp. 5 - 11, 2019.

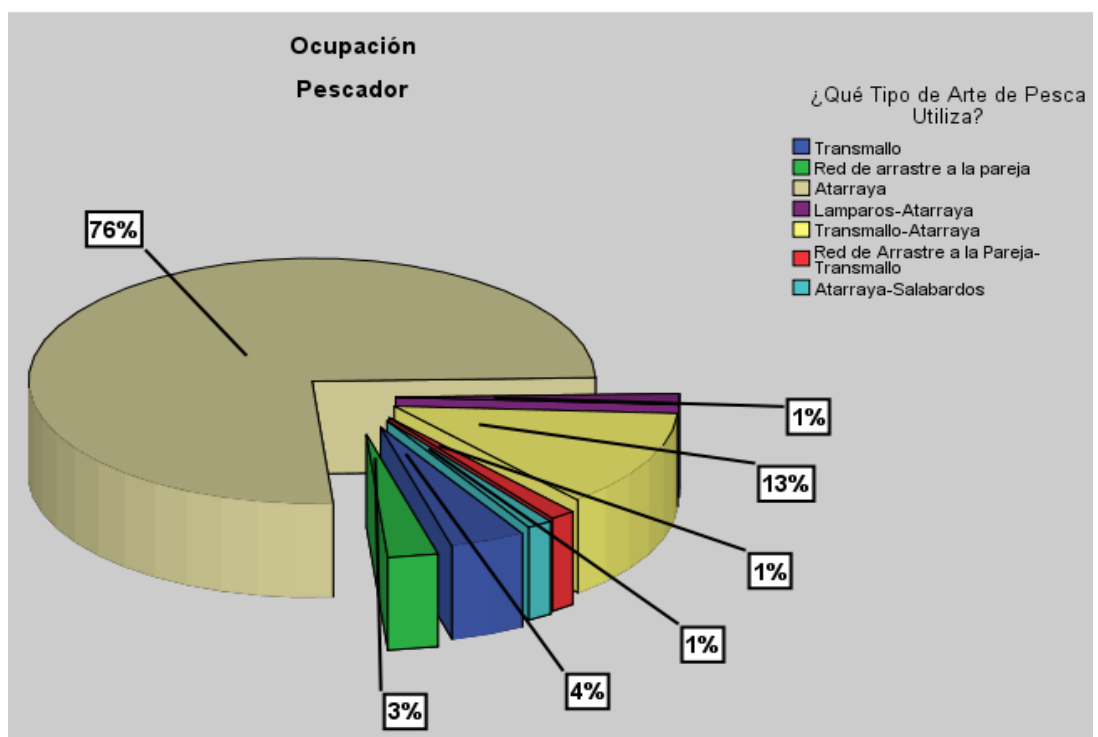

Figura 2. Tipos de arte de pesca utilizadas en el embalse El Guajaro.

Fuente: Los autores.

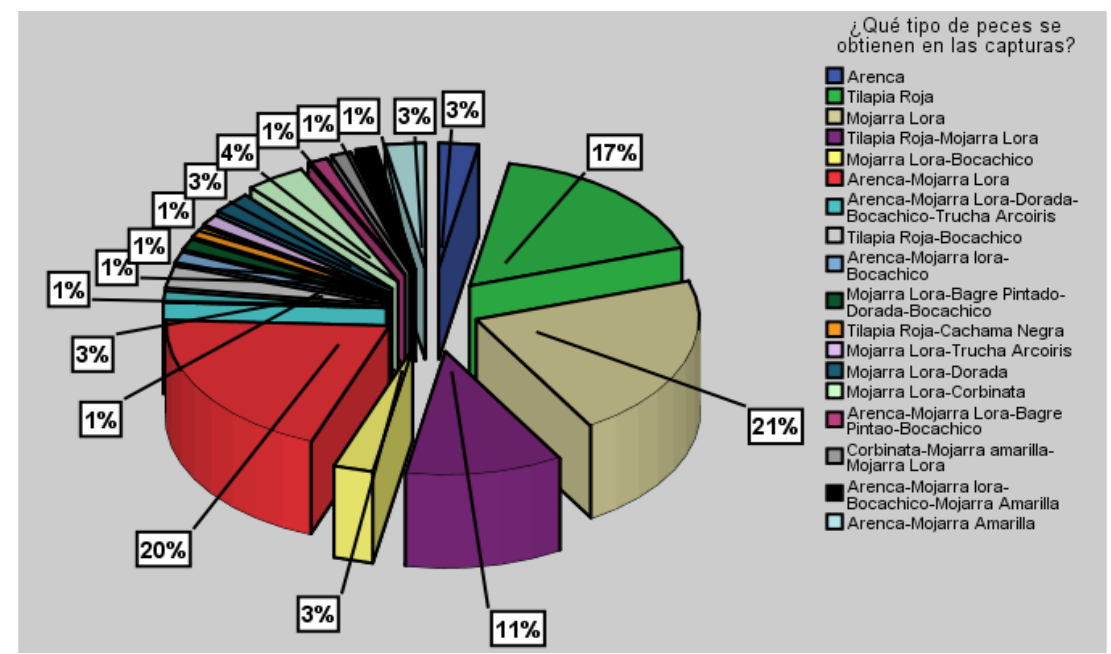

Figura 3. Tipos de peces atrapados en el embalse El Guajaro.

Fuente: Los autores.

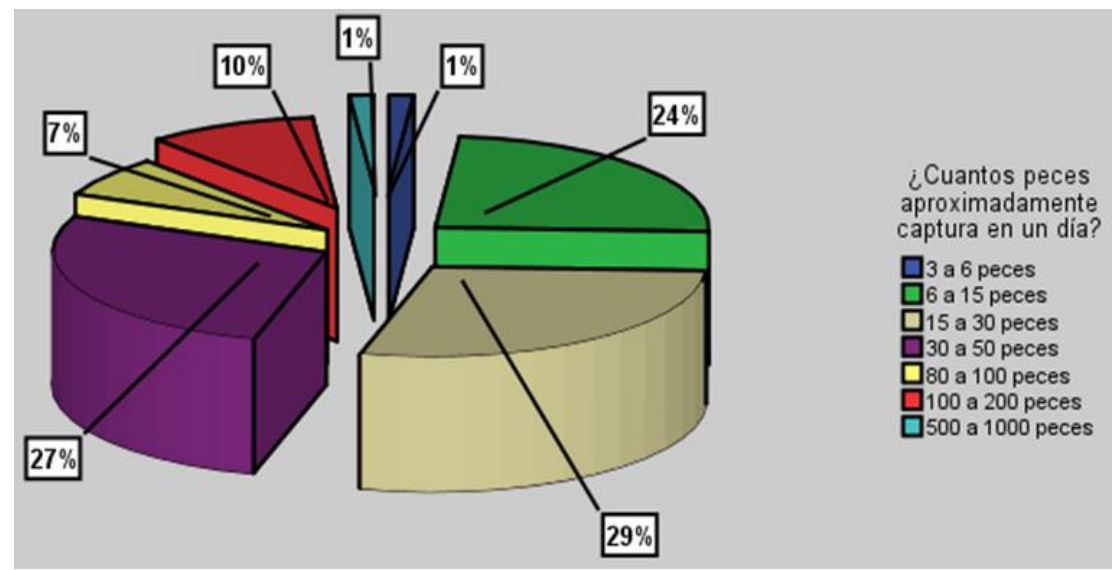

Figura 4. Cantidad de peces captura por día.

Fuente: Los autores. 


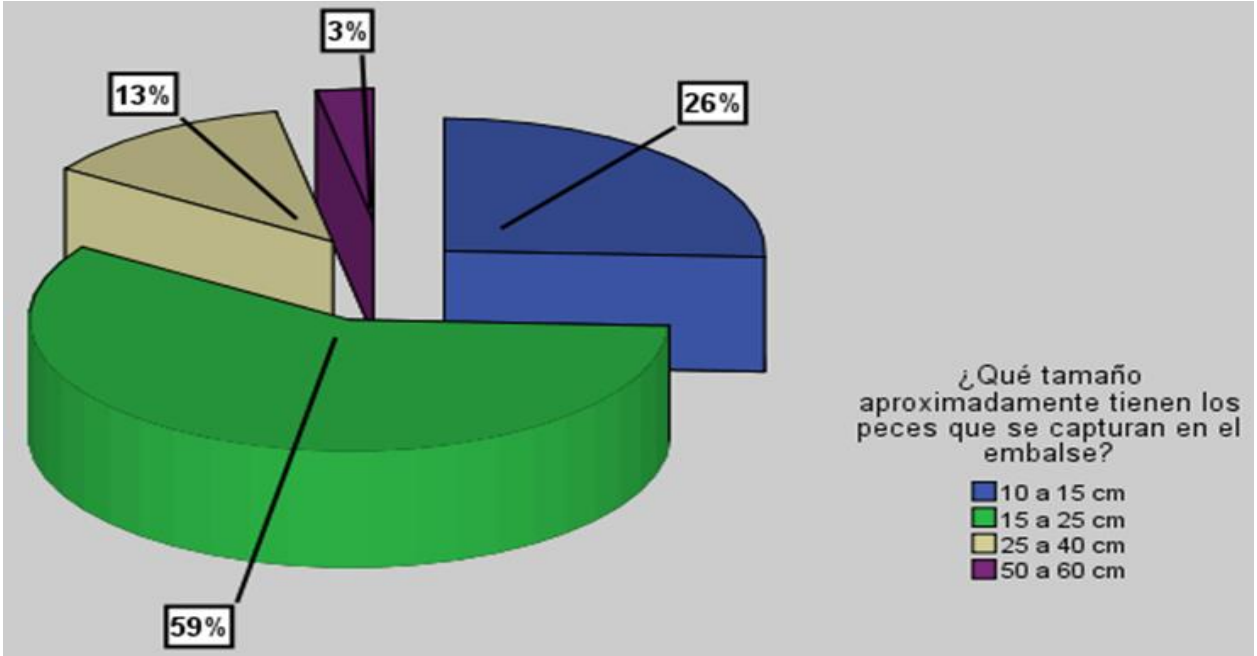

Figura 5. Tamaño de peces capturados.

Fuente: Los autores.

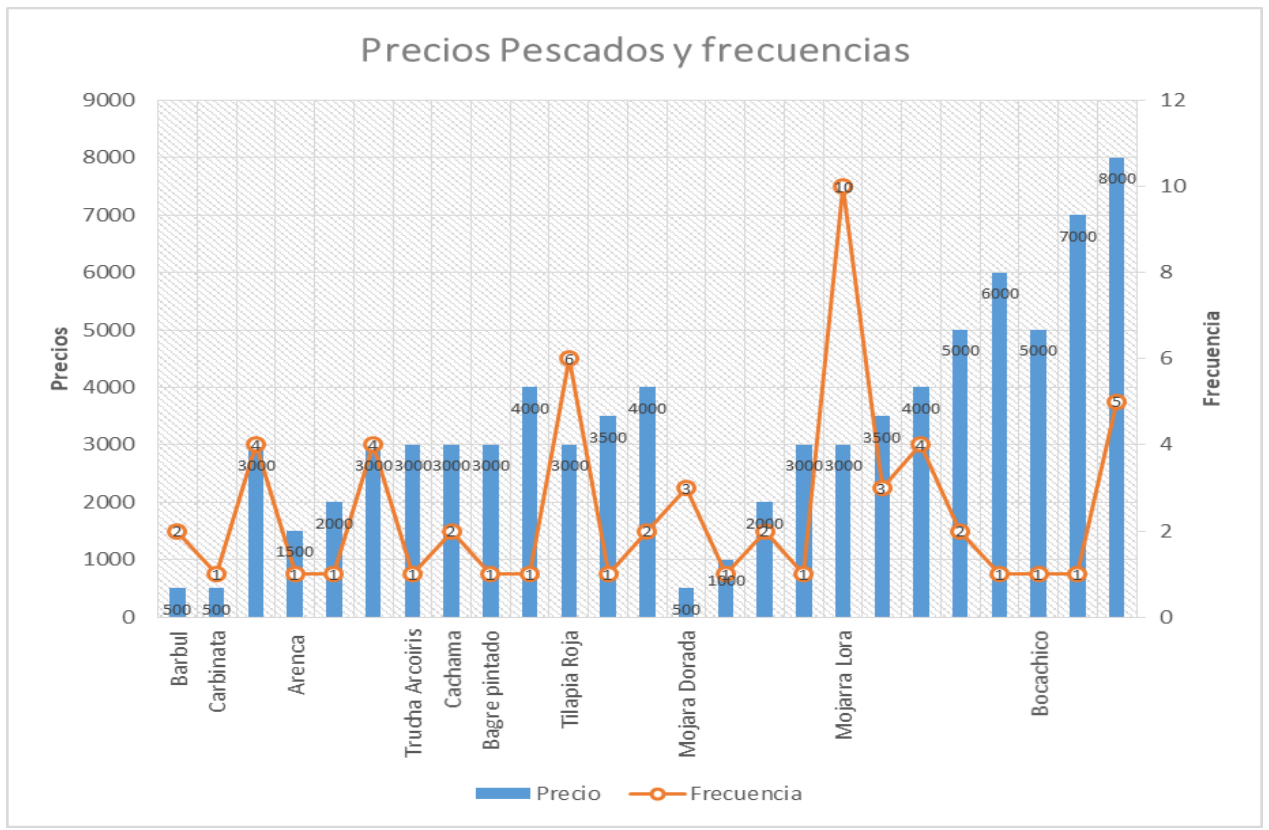

Figura 6. Precios de peces y frecuencias.

Fuente: Los autores.

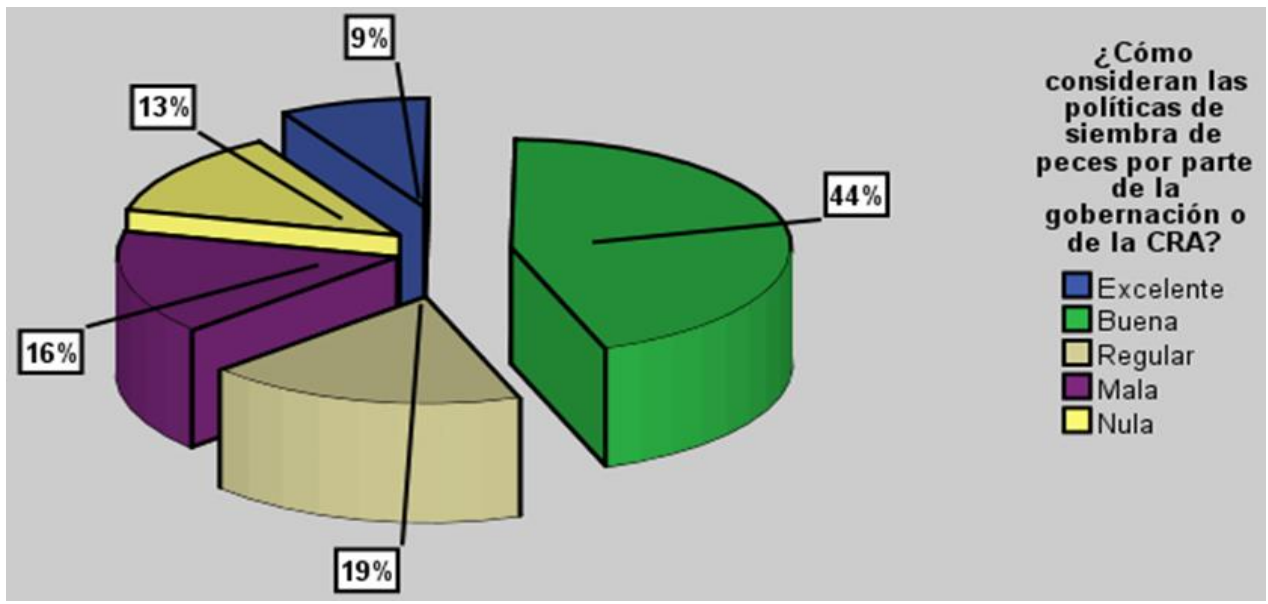

Figura 7. Políticas CRA y Gobernación.

Fuente: Los autores. 
Los peces oscilan en tamaños entre los 10 y los 60 centímetros. La mayor representatividad en el tamaño de los peces la tiene el grupo que va entre los 15 y los 25 centímetros, teniendo cerca de un $59 \%$ de la muestra evaluada. Se obtuvo también que los peces con medidas entre los 10 y los 15 centímetros tienen una participación del 26\%; y los que miden entre 25 y $60 \mathrm{~cm}$, de un $16 \%$. En consecuencia, el comercio muestra de los productos en tendencia por ellos se muestra los precios de peces y frecuencias. Figura 6

Los productos con menor costo son el barbul y la carbinata (\$500), y el de mayor costo es el bocachico (\$8.000). La mayor tendencia se encuentra en la mojarra lora (\$3.000), siendo este el producto más comercializado en ese estándar de precio. Por ello las políticas de siembra que se consideran son las establecidas por la CRA y la Gobernación. Figura 7

Se considera que el $53 \%$ de las políticas implementadas para el sector, aplicadas por la CRA y la Gobernación del Atlántico, tienen resultados positivos

\section{Conclusiones}

Se evidencia el uso de artes prohibidas para el desarrollo de la pesca, esto debido a la falta de control y regulación por parte de la autoridad ambiental y las pocas acciones policivas.

Se encontró que los tamaños de los peces oscilan entre los 10 y los 60 centímetros, la mayor tendencia de tamaño se encuentra entre los 15 y 25 centímetros, esto probablemente a la reducción de la producción propia del cuerpo de agua, debido, quizás, a la contaminación y a las políticas erradas de la CRA en el repoblamiento del embalse.

En promedio los ingresos por pescador no superan el salario mínimo actual vigente, además, teniendo en cuenta los costos de los insumos para el desarrollo de la pesca y en algunos casos el alquiler de los equipos para el desarrollo de esta actividad hace que estos disminuyan considerablemente.

La aceptación de las políticas públicas solo se encuentra por encima del 50\%. Se encontró, además, desconocimiento de las acciones que desarrollan entidades como la CRA y la
Gobernación del Atlántico, por lo cual se sugiere mayor dialogo con dichos actores.

\section{Referencias}

Aunap-unimagdalena, 2014. Caracterización de los principales artes de pesca de Colombia y reporte del Consolidado del tipo y número de artes, embarcaciones y uep's empleadas por los pescadores vinculados a la actividad pesquera. Contrato de prestación de servicios no. 190, suscrito entre la autoridad nacional de acuicultura y pesca y la universidad de la magdalena. Santa marta y Bogotá. $72 p$

Baigún, C. R. (2013). Manual para la gestión ambiental de la pesca artesanal y las buenas prácticas pesqueras en la cuenca del río Paraná, Argentina. Fundación Humedales/Wetlands International. Buenos Aires, Argentina.

Estrella Arellano, C., Guevara Carrasco, R., \& Palacios León, J. (1998). Informe estadístico de los recursos hidrobiológicos de la pesca artesanal por especies, artes, caletas y meses durante el primer semestre de 1998.

García, C. B. (2010). Conocimiento tradicional: lo que los pescadores artesanales del Caribe colombiano tienen para decirnos. PanAmerican Journal of Aquatic Sciences, 5(1), 7890.

Herrera, O., \& Cortés, O. G. (2012). Recursos hidrobiológicos del departamento del atlántico: problemática y alternativas de desarrollo. Dugandia, 4(1).

Izzo, A., Isla, M., Salvini, L., Bartozzetti, J. D., Garcia, J. C., Roth, R. R., ... \& Ércoli, R. (2000). Artes y métodos de pesca desarrollados en el Canal de Beagle, Tierra del Fuego, Argentina.

Marín A., W. (2007). Cultura y modernización de la pesca artesanal en Chile: Adaptaciones, cambios e hibridaciones en una caleta de algueros. Revista Mad, (17), 113-143. doi:10.5354/07180527.2011.13941

Oyaga Martínez, R. F. (2013). Realidades Ambientales de los Cuerpos de Agua del departamento del Atlántico, Colombia. 
Puentes, V., Polo, C. J., Roldán, A. M., \& Zuluaga, P. A. (2014). Artes y métodos de pesca en Colombia. Serie Recursos Pesqueros de Colombia. Autoridad Nacional de Acuicultura y Pesca-AUNAP. Bogotá: Conservación Internacional, 216. 